\title{
The Past, Present and Future Situation of Mixed Martial Arts (MMA) in China
}

Xin $\mathrm{CHEN}^{*}$, \& Guodong ZHANG

College of Physical Education, Southwest University, Chongqing, China

\section{5th IMACSSS World Scientific Congress Abstracts, Rio Maior (Portugal), October 6-8}

Section: Adaptative MA\&CS

Type: Oral communication

\section{Introduction}

China, with a history of more than 5,000 years, has created uncountable splendid civilizations. Chinese folk martial arts (MA) contests and challenge competitions in Song Dynasty (960-1279 A.D.) are seen as the rudiment of the unarmed MMA (Wang \& Li, 2014). In 1999, Andy, a Chinese American, started to promote the development of MMA in China (Ben, 2012). The introduction of MMA provides an opportunity for the contests between Chinese athletes and top-class ones in the world. What's more, during those contests, gifted and potential young athletes got greatly improved, presenting us a bright future of the new generation with strong competitive force. From its features, MMA is regarded as not only an activity which can improve the athletes, but also the master of commercial factors such as visual impact. Additionally, much similar to traditional Chinese challenge competitions, MMA is capable of meeting the needs of many Chinese people. Therefore, in the field of Chinese MA, promoting the MMA is of great significance for the development of the fighting arena industry. Now MMA, as a global sport activity, is still in the initial stage, with quite few related researches. As a result, Chinese MMA lacks scientific and appropriate theoretical guidance, which leads to the obstacles for the spread and promotion of MMA in China. This paper reviews the origin, analyses and summarizes the present situation and problems, and finally prospects a rational future direction of MMA in China, aiming at providing a reference for the further development of MMA in China.

\section{Methodology}

(1) Literature data method adopted in the research: the study was conducted through reading and sorting authoritative literature and articles in CNKI and EI database including academic work on sport culture, sport industry, sport broadcasting and sport professionalization and collecting all kinds of relevant literature about MMA at home and abroad. (2) Observational method: I participated in the training of MMA athletes myself. Therefore, I experienced and knew the practical situation of MMA athletes both in their training and in their lives, and consulted relevant questions. All these information were elaborated and summarized in the end. Besides, a lot of materials were attained by watching videos and observing image-text of various MMAs online and watching MMAs on the spot at home and abroad. (3) Interviewing method: More than one way was used to gather the real development status of MMA, including telephone interview and field interview with experts and athletes who contacted and spread MMA at the earliest, coaches of Chinese MMA Club and experts in physical colleges. I also interviewed the experts and scholars specialized in sport sociology and sport economics then, to fully comprehend the development status of MMA in different fields and the advantages and disadvantages brought to them.

\section{Results}

Previously, Chinese nation has been martial since ancient times, elucidating warrior spirit from the Emperor $\mathrm{Wu}$ of Han Dynasty to Bruce Lee. In Chinese history, MMA developed from the early 
challenge competition in Song Dynasty and folk MA contests. In 1999, Andy, a Chinese American, started to bottom and develop MMA in China. One problem at that time was that MMA was hard for Chinese massive to accept because of its security concerns. When it comes to MMA, people immediately relate it with blood and cruelty. To the untrained eyes, MMA seems quite dangerous. However, MMA actually is a sports activity with high security and also keeps favorable records. In 2002, the first MMA club, named “E Tong Legion” (恶童军团) was founded, and the first MMA competition in China was then launched in a martial club at Wanshou Road, Beijing. Long before 1988, Andy had established a training group of Brazilian jiu-jitsu where jiu-jitsu amateurs were invited to teach people without any charge as the predecessor of E Tong Legion in Beijing which is the initial stage of China's modern MMA. At present, MMA in China shows a tendency for robust and positive development. Many fight clubs have already set up MMA courses and some clubs even organized professional MMA clubs. Meanwhile, the MA management center of General Administration of Sport in China has also built special organizations for MMA referees training. With the support and joint efforts of all walks of life, MMA in China is moving to a new stage. Nonetheless, there are still some problems in the development of MMA in China. Firstly, the contests organization of MMA is still in a maintenance condition. As the organizers in the early stage just organized MMA amateurs to compete without considering market development and rigorous competition operation process, they failed to make a difference to Chinese MMA market development. Secondly, there is a lack of MMA athletes, especially sports stars in this field. There is also a gap between different ages of athletes. Especially for bottom MMA athletes, they could not gain enough money to support their lives. As a result, the training was affected, which resulted in a great shortage of MMA reserve forces. Thirdly, the incapability of the MMA referees is also a big problem, demand of systematic and professional training. Only professional MMA judges can guarantee competitions to go on smoothly and protect athletes better. Fifthly, athletes' cultivation does not form a certain scale. Lastly, the supervision and management of related departments of MMA in China are not enough. The reasons for the above problems are multi-faceted, but what matters is to find the root causes. It is necessary for us to contrast the main influence factors between domestic and foreign MMA development so that we can find out our own shortages and the directions we strive for. Despite of existing problems, it is true that MMA shows a developing tendency on the Chinese fighting contests in the future. In the future, with the entering of American UFC contest, Chinese MMA athletes are provided with the opportunity to communicate with and learn from top-class MMA athletes in the world, which lays a solid foundation for the future development of MMA in the whole world. It is definite that MMA in China will surpass many other sports activities in the future with a shining prospect. My viewpoints on better development of Chinese MMA are as follows: first, strengthening the publicity and construction of MMA social environment. Second, strengthening the supervision and specification of MMA market subject behaviors. Third, setting up platforms to facilitate the communication of MMA subject behaviors. Fourth, building and improving cultivation mechanism of MMA athletes.

\section{Discussion and conclusion}

In the past, MMA in China derived from the MA contests and challenge competition, lacking the fusion and application of multiple MA techniques, which hindered its blossom. Now MMA in China is developing very strongly. Although it is a long way for the development of MMA in China together with many possible difficulties and problems, what we just need do is to compete with western countries with an attitude of promoting the Chinese MA and to enhance our overall MMA ability fundamentally. MMA is the new product following the fast development of the MA in the world. It is also regarded as a necessary road for Chinese MA entering the world stage and become the one in the global MA family. Therefore, MMA in China has extensive room for progression.

\section{References}

Ben, K. (2012). Exploring the origins and passing stories of MMA in China. Boxing \& Fight, 11(6), 3741

Wang, H., \& Li, J. (2014). The indispensable discussion on the carrying out of MMA in China. Chinese Wushu Research, 4(7), 93-96

Key words: Mixed Martial Arts; Chinese martial arts; combat sports; martial arts development. 